\title{
Multiple valued input generalised Reed-Muller forms
}

\author{
I. Schäfer \\ M.A. Perkowski
}

Indexing terms: Multiple valued inputs, Programmable devices, Reed-Muller expansion

\begin{abstract}
The concept of canonical multiple valued input generalised Reed-Muller (MIGRM) forms is introduced. The MIGRM is a direct extension of the well known generalised ReedMuller (GRM) forms to the logic with multiple valued inputs. The concept of the polarity of a GRM form is generalised to the polarity matrix of a MIGRM form. A tabular pattern-matching method is presented for the calculation of a MIGRM form. The MIGRM transform has been implemented for further investigations of such forms and their comparison with other circuit realisations.
\end{abstract}

\section{Introduction}

The concept of a fixed-polarity generalised Reed-Muller (GRM) form $[1,2]$ of an $n$-input Boolean function has been studied extensively in the literature. One reason for studying such forms is that, for each of the $2^{n}$ polarities, they are canonical, which has several applications in both theory and practice. They have been intensively studied for better understanding of the canonical representations of switching functions [2-5]. As is well known, the circuits corresponding to Reed-Muller and GRM forms have excellent design-for-test properties [6-11]. Finally, GRMs have applications in signal coding and image processing [12].

In recent years a logic with multiple valued inputs has been introduced with applications in synthesis of PLAs with decoders and function generators [13-15], multilevel logic synthesis and factorisation $[16,17]$. A multiple valued input, binary output function can be readily implemented with currently available digital circuits, while a real multiple valued function cannot. The concept of multiple valued input Exclusive-OR sum-of-products (ESOP) expressions has been presented in Reference 18.

This paper introduces the counterpart of GRMs for the logic with multiple valued inputs and binary outputs. We will call such forms multiple valued input generalised Reed-Muller forms (MIGRMs). The counterpart to the RMs, the restricted multiple valued input generalised Reed-Muller forms (RMIGRMs), have been introduced in Reference 19.

A motivation for the investigation of MIGRM forms is that the concept of the AND-EXOR PLA [20], which

Paper 9102E (C2), first received 18th July 1991 and in revised form 6th July 1992

The authors are with the Department of Electrical Engineering, Portland State University, PO Box 751, Portland, OR 97207-0751, USA

IEE PROCEEDINGS-E, VOL. I39, NO. 6, NOVEMBER 1992 is used for the realisation of ESOPs, can be modified to MIGRMs. The AND-EXOR PLA with two-input, fouroutput input decoders [20] is used for the multiple valued input, binary output exclusive sum-of-products (MIESOP) expansions. Similarly, it can be used for the MIGRMs. Moreover, if the RMIGRM forms [19] are applied, the AND/EXOR needs only two-input, threeoutput input decoders. Although one gains $25 \%$ on one dimension of the PLA, one can lose in the other dimension, as the number of terms is usually larger in a GRM and a RMIGRM or MIGRM than in an ESOP or MIESOP of the same function. But the GRM and MIGRM have the advantage of excellent testability properties which have been proven for the strict ReedMuller forms [10] and are extendable for the GRMs and MIGRMs.

The existence of new programmable devices such as the Xilinx LCA 3000 , the 1020 series from Actel, or the LHS501 from Signetics allows for the direct implementation of the form introduced here. For instance, in Xilinx LCA 3000 devices, every module realisation of a Boolean function of five variables has the same cost and speed. The use of EXORs is then reasonable, as they are more powerful than the inclusive gates. It has been proven that the circuits with EXOR gates have lower worst-case complexity than the circuits which use only inclusive gates [15]. Moreover, EXOR-based circuits such as MIGRMs, GRMs or ESOPs have much better testability properties than SOPs.

This paper presents the basic research on MIGRMs for completely specified multiple valued input, binary multioutput functions. We introduce the general concept of a tabular pattern matching method to calculate the MIGRM. Section 2 presents the theory of the MIGRM forms, and Section 3 gives the algorithm for the MIGRM transform. Section 4 illustrates the transformation of the multiple valued input, multioutput SOP (sum-of-products) function of a two-bit adder to a MIGRM form.

2 Canonical multiple valued input, binary output generalised Reed-Muller forms

Canonical forms have already been proposed for multiple valued input, multiple valued output functions [21, 22]. The $m$-Reed-Muller canonical ( $m$-RMC) forms [22] are obtained from the truth vector or the SOP representation and the generalisation of the Boolean difference to multiple valued logic. However, such forms cannot be realised

The work presented in this paper was partially supported by NSF grant MIP-9110772 
with available digital circuits. Therefore, the MIGRM introduced here is based on the multiple valued input, binary output logic.

Definition 1: A multiple valued input, completely specified binary output function ( $m v$ function for short) is a mapping $f(X)=f\left(X_{1}, \quad X_{2}, \ldots, \quad X_{n}\right): \quad R_{1} \times R_{2} \times \cdots$ $\times R_{n} \rightarrow B$, where $X_{i}$ is a multiple valued variable that takes the values from the set $R_{i}=\left\{0,1, \ldots, p_{i}-1\right\}$ where $p_{i}$ is the number of values of the variable, and $B=\{0,1\}$.

Definition 2: A literal of the multiple valued input variable $X_{i}$, denoted by $X_{i}^{S_{i}}$, is defined as

$$
X_{i}^{S_{i}}= \begin{cases}1 & \text { if } X_{i} \in S_{i} \\ 0 & \text { if } X_{i} \notin S_{i}\end{cases}
$$

A literal of an $m v$ variable with a single value will be called a single-valued literal. A product of literals, $X_{1}^{S_{1}}$, $X_{2}^{s_{2}}, \ldots, X_{n}^{s_{n}}$ is referred to as a product term (also called term for short)

The approach presented here to generate a MIGRM makes use of spectral methods similar to the ones introduced in References 19 and 23. First the concept of polarity is introduced for a multiple valued literal. Then it is shown how it is further applied for a product term of multiple valued literals.

2.1 Concept of polarity for a multiple valued variable The concept of the polarity of a singular multiple valued literal is given by the following theorem.

Theorem 1: Multiple valued literal $X_{i}^{S_{i}}$, where $S_{i} \subseteq R_{i}[0$, $\left.1, \ldots, p_{i}-1\right]$ and $X_{i}^{S_{i}} \subseteq R_{i}$, can be represented by $p_{i}$ polarity literals $P_{i}^{T_{i}}$ with the set of truth values $T_{i} \subseteq R_{i}$. The values of the $p_{i}$ polarity literals form the row vectors of the orthogonal $p_{i} \times p_{i}$ polarity matrix $P_{i}$.

Proof: A property of an orthogonal $m \times m$ matrix $O(m$ is an arbitrary natural number) with the elements $o_{i j} \in(0,1)$ is that any vector $U\left(u_{0}, u_{1}, \ldots, u_{m-1}\right)$ with $u_{j} \in(0,1)$ can be represented by a superposition (that is, performing a bit-by-bit EXOR operation) of row vectors $O_{i}$ of the matrix $O$. Thus, any set $S$ of truth values $S \subseteq R=\{0,1$, $\ldots, p-1\}$ of a literal $X^{S}$ can be represented by a superposition of the values from the orthogonal set of $p$ truth values $T \subseteq R=\{0,1, \ldots, p-1\}$, where $T^{\prime}$ is the $r$ th row vector of the orthogonal $p \times p$ matrix $P$.

A form according to theorem 1 is canonic because the polarity matrix $P$ is orthogonal.

Example 1: To illustrate theorem 1, all possible sets of truth values $S \subseteq R=\{0,1,2\}$ of a three-valued literal $X^{s}$ are calculated from the chosen $3 \times 3$ orthogonal matrix shown in Fig. 1. In Table 1 the calculations of all possible

$P=\left[\begin{array}{lll}0 & 1 & 1 \\ 0 & 1 & 0 \\ 1 & 0 & 0\end{array}\right]=\left[\begin{array}{l}T_{1} \\ T_{2} \\ T_{3}\end{array}\right]$

Fig. 1 Example of a $3 \times 3$ orthogonal matrix sets of truth values $S$ by exoring the rows of the orthogonal matrix $P$ (Fig. 1) are given, where the rows are denoted by $T_{1}, T_{2}$ and $T_{3}$.

Table 1 : All possible superpositions of polarity literals

\begin{tabular}{lll}
\hline Truth values $S$ & Binary code & Superposition \\
\hline$\{2\}$ & 001 & $T_{1} \oplus T_{2}$ \\
$\{1\}$ & 010 & $T_{2}$ \\
$\{1,2\}$ & 011 & $T_{1}$ \\
$\{0\}$ & 100 & $T_{3}$ \\
$\{0,2\}$ & 101 & $T_{1} \oplus T_{2} \oplus T_{3}$ \\
$\{0,1\}$ & 110 & $T_{2} \oplus T_{3}$ \\
$\{0,1,2\}$ & 111 & $T_{1} \oplus T_{3}$ \\
\hline
\end{tabular}

Definition 3: The matrix $P_{i}$ introduced in theorem 1 is called the polarity matrix or, for short, the polarity of a multiple valued variable $X_{i}$. The $r$ th row vector $T_{i}^{r}$ of the matrix $P_{i}$ is the binary representation of the polarity literals $P_{i}^{T_{i}{ }^{r}}\left(P_{i}^{r}\right.$ for short).

For the representation of the polarity literals $P_{i}^{r}$, nonorthogonal matrices can also be used. Thus, theorem 1 can be generalised to the following lemma.

Lemma 1: Instead of the orthogonal matrix $P$, defined in theorem 1, any set of vectors $T^{r}$ can be used for the polarity literals $P^{r}$ if, by exoring of those literals, every possible set $S \subseteq R=\{0,1, \ldots, p-1\}$ of a variable $X^{s}$ can be generated. Thus, there is more than one way to create an $m v$-literal $X_{i}^{S}$ out of the polarity literals given by the nonorthogonal matrix. AND-EXOR expressions created with such polarity literals are no longer canonical forms.

The MIESOP expressions $[18,20]$ are examples of expressions generated by polarity literals as described in lemma 1.

Table 2 summarises the notation presented in definition 3 and theorem 1. In the first row of Table 2, the multiple valued literals $X_{i}^{s_{i}}$ of a function $F\left(X_{1}, X_{2}, \ldots\right.$, $X_{n}$ ) are shown. In the second row, the sets of truth values $P_{i}$ for those literals are given. Next, the corresponding polarity literals $P_{i}^{r}$ are shown, where the $r$ stands for the values represented by the $T_{i}^{r}$ vector of the matrix $P_{i}$. Finally, Fig. 2 illustrates two polarity matrices $P_{i}$ consisting of the value vectors $T_{i}^{r}$.

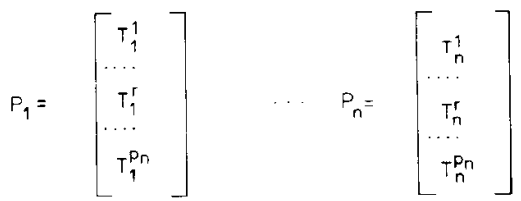

Fig. 2 Description of polarity matrices

\subsection{MIGRM forms}

Before the MIGRM forms are formally defined, let us consider a simple example of such a form.

Example 2: The two-variable $m v$ function $F_{1}\left(X_{1}, X_{2}\right)=$ $X_{1}^{023} X_{2}^{01}$, where $X_{1}$ is four-valued and $X_{2}$ is threevalued, is used to show how to calculate the MIGRM

Table 2: Notation for MIGRM

\begin{tabular}{|c|c|c|c|c|}
\hline $\begin{array}{l}m v \text { literal } \\
\text { set of truth values } \\
\text { polarity literals } \\
\text { polarity matrix }\end{array}$ & $\begin{array}{l}X_{1}^{s_{1}} \\
R_{1}=\left(0,1, \ldots, P_{1}-1\right) \\
P_{1}^{1} \ldots \ldots P_{1}^{r}, \ldots, P_{1}^{o_{1}} \\
P_{1}^{1}\end{array}$ & $x_{2}^{s_{2}}$ & $\begin{array}{l}X_{i}^{s_{i}} \\
R_{i}=\left(0,1, \ldots, p_{i}-1\right) \\
\cdots \\
P_{i}\end{array}$ & $\begin{array}{l}X_{n}^{s_{n}} \\
R_{n}=\left(0,1, \ldots, P_{n}-1\right) \\
P_{n}^{1}, \ldots, P_{n}^{\prime}, \ldots, P_{n}^{D_{n}} \\
P_{n}\end{array}$ \\
\hline
\end{tabular}


form for the polarity given in Fig. 3. The variable $X_{2}^{01}$ can be represented by the superposition of the polarity literals $P_{2}^{1} \oplus P_{2}^{3}$, where for $P_{2}^{1}$ the subscript 2 indicates the corresponding $m v$ literal $X_{2}$ and the superscript 1 denotes the values $T_{2}^{1}$ for the $m v$ literal $X_{2}$. The natural method to perform the transformation would seem to be by an EXOR-term multiplication, i.e.

$$
\begin{aligned}
X_{1}^{023} X_{2}^{01} & =\left(P_{1}^{1} \oplus P_{1}^{3} \oplus P_{1}^{4}\right)\left(P_{2}^{1} \oplus P_{2}^{3}\right) \\
& =\left(1 \oplus P_{1}^{3} \oplus P_{1}^{4}\right)\left(1 \oplus P_{2}^{3}\right) \\
& =1 \oplus P_{1}^{3} \oplus P_{1}^{4} \oplus P_{2}^{3} \oplus P_{1}^{3} P_{2}^{3} \oplus P_{1}^{4} P_{2}^{3}
\end{aligned}
$$

In example 2, the multiplication is applied to obtain the MIGRM form from the polarity literals. To perform the

$$
\begin{aligned}
& A_{1}=\left[\begin{array}{l}
1111 \\
0101 \\
0011 \\
0111
\end{array}\right]=\left[\begin{array}{l}
T_{1}^{1} \\
T_{1}^{2} \\
T_{3}^{3} \\
T_{1}^{4}
\end{array}\right] \\
& A_{2}=\left[\begin{array}{l}
111 \\
100 \\
001
\end{array}\right]=\left[\begin{array}{l}
T_{2}^{1} \\
T_{2}^{2} \\
T_{2}^{3} \\
2
\end{array}\right]
\end{aligned}
$$

Fig. 3 Polarity matrices

multiplication of the polarity literals shown there, the MIGRM form will be described as a spectrum $M$. A tabular pattern matching method between the indices of the spectral coefficients and a product term will be introduced to calculate the final MIGRM from the polarity representation of the literals $X_{i}^{S_{i}}$

The following definition extends the concept of the GRM forms for Boolean functions to the concept of MIGRM forms for $m v$ functions.

Definition 4: The multiple valued input, binary output generalised Reed-Muller (MIGRM) form of a single output function $F\left(X_{1}, X_{2}, \ldots, X_{n}\right)$, where $X_{i}, i=1,2$, $\ldots, n$ are the $m v$ literals, is defined as follows

$$
\begin{aligned}
F\left(X_{1}, X_{2}, \ldots, X_{n}\right)= & a_{0} P_{1}^{1} P_{2}^{1} \cdots P_{n}^{1} \\
& \oplus a_{1} P_{1}^{1} P_{2}^{1} \cdots P_{n}^{2} \\
& \oplus \cdots \oplus a_{p_{n}} P_{1}^{1} P_{2}^{1} \cdots P_{n}^{p_{n}} \\
& \oplus \cdots \oplus a_{t} P_{1}^{p_{1}} P_{2}^{p_{2}} \cdots P_{n}^{p_{n}}
\end{aligned}
$$

where $a_{i} \in\{0,1\}$ and $t=\prod_{i=0}^{n-1} p_{i}$; the other notation follows the one in Table 2. For a set of functions $F_{f}\left(X_{1}\right.$ $X_{2}, \ldots, X_{n}$ ) we obtain

$$
\begin{aligned}
F_{j}\left(X_{1}, X_{2}, \ldots, X_{n}\right)= & a_{0_{j}} P_{1}^{1} P_{2}^{1} \cdots P_{n}^{1} \\
& \oplus a_{1 j} P_{1}^{1} P_{2}^{1} \cdots P_{n}^{2} \\
& \oplus \cdots \oplus a_{p_{n j}} P_{1}^{1} P_{2}^{1} \cdots P_{n}^{p_{n}} \\
& \oplus \cdots \oplus a_{t_{j}} P_{1}^{p_{1}} P_{2}^{p_{2}} \cdots P_{n}^{p_{n}}
\end{aligned}
$$

Definition 5: The polarity of a MIGRM form is the vector of polarity matrices describing the polarity for each multiple valued literal in the form.

The above definitions can be described with the terminology of spectral techniques as shown in Reference 24 for the GRM form.
Definition 6: The MIGRM given by definition 5 can be represented by the spectrum $M$, where the index of a spectral coefficient $M_{P_{1}^{r} \ldots P_{n}^{r}}$ corresponds to the terms of polarity literals $P_{1}^{r} \cdots P_{n}^{r}$ in eqns. 1 and 2 . Those terms represent the standard trivial functions [23] of the MIGRM spectrum.

Example 3: The MIGRM of the function $F_{1}\left(X_{1}, X_{2}\right)=$ $X_{1}^{023} X_{2}^{01}$ (see example 2) can be represented by its spectrum $M$. Fig. 4 gives the standard trivial functions of the

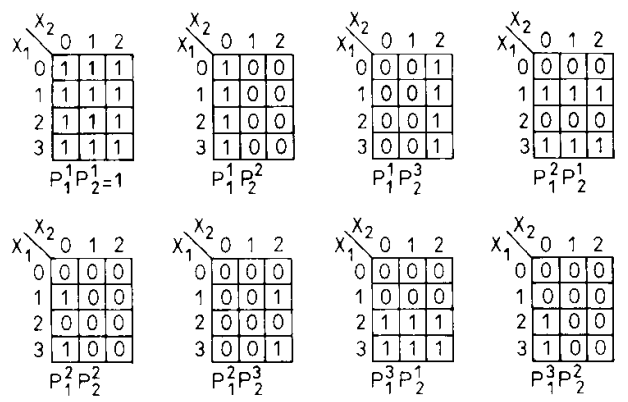

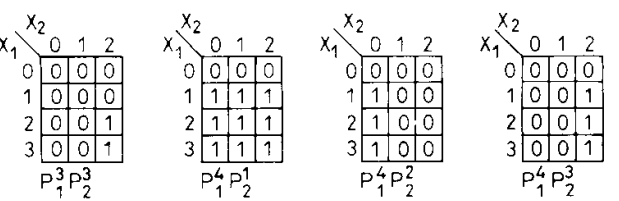

Fig. 4 Standard trivial functions for polarities of variables $X_{1}$ and $X_{2}$ specified in Fig. 3

above product term for the polarity from Fig. 3. For a comparison of the product $X_{1}^{023} X_{2}^{01}$ with the standard trivial functions in Fig. 4, the map of this product is shown in Fig. 5.

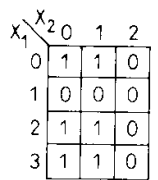

Fig. 5 Map of function $F\left(X_{1}, X_{2}\right)=X_{1}^{023} X_{2}^{01}$ from examples 2 and 3

The new expression can be represented now in the

\begin{tabular}{|c|c|c|c|c|c|c|c|c|c|c|c|c|}
\hline Coefficient & $M_{\rho_{1}^{1} \rho_{2}^{1}}$ & $M_{\rho\} \rho_{2}^{2}}$ & $M_{\rho \rho_{1}^{3} \frac{3}{2}}$ & $M_{p_{1}^{\bar{z}} p_{2}^{1}}$ & $M_{P_{1}^{2} p_{2}^{2}}$ & $M_{p_{1}^{2} \rho_{\frac{3}{2}}^{3}}$ & $M_{P_{1}^{3} P \frac{1}{2}}$ & $M_{p_{1}^{3} \rho_{2}^{2}}$ & $M_{p_{1}^{3} p_{2}^{3}}$ & $M_{P_{1}^{4} p_{2}}$ & $M_{P_{1}^{4} \rho_{2}^{2}}$ & $M_{P_{1}^{4} \rho_{2}}$ \\
\hline value & 1 & 0 & 1 & 0 & 0 & 0 & 1 & 0 & 1 & 1 & 0 & 1 \\
\hline
\end{tabular}
form of spectral coefficients (see Table 3), where the indices correspond to the standard trivial functions. The same result as in example 2 has been obtained

$$
X_{1}^{023} X_{2}^{01}=1 \oplus P_{1}^{3} \oplus P_{1}^{4} \oplus P_{2}^{3} \oplus P_{1}^{3} P_{2}^{3} \oplus P_{1}^{4} P_{2}^{3}
$$

The reader may wish to verify this form by exoring the corresponding maps from Fig. 4 to obtain the map from Fig. 5. The algorithm for calculating this form will be given in Section 3.

The 'coefficient' row of Table 3 gives all possible spectral coefficients, where the MIGRM terms represent all the indices for a general function where the arguments are a four-valued literal $X_{1}$ and a three-valued literal $X_{2}$.

Table 3: Spectrum of function $F,\left(X_{1}, X_{2}\right)$ 
In the 'value' row, a ' 1 ' indicates that the term represented by the index of the spectral coefficient in the same column is present in the MIGRM form. In Table 3, the terms $M_{P_{1}^{1} P_{2}^{\frac{1}{2}}}, M_{P_{1}^{1} P_{2}^{3}}, M_{P_{1}^{3} P_{2}^{\frac{1}{2}}}$, and $M_{P_{1}^{4} P_{2}^{1}}$ are identical to 1 , $P_{2}^{3}, P_{1}^{3}$, and $P_{1}^{4}$ because $P_{1}^{1}=P_{2}^{1}=1$.

The final MIGRM form is now obtained by substitution of the polarity literals for their multiple valued literal representation according to the polarity matrices. Thus

$$
\begin{aligned}
X_{1}^{023} X_{2}^{01} & =1 \oplus P_{2}^{3} \oplus P_{1}^{3} \oplus P_{1}^{3} P_{2}^{3} \oplus P_{1}^{4} \oplus P_{1}^{4} P_{2}^{3} \\
& =1 \oplus X_{2}^{2} \oplus X_{1}^{23} \oplus X_{1}^{23} X_{2}^{2} \oplus X_{1}^{123} \oplus X_{1}^{123} X_{2}^{2}
\end{aligned}
$$

Example 4 illustrates the transformation of multioutput functions consisting of more than one product term. The transformation is based on the input function being in ESOP form or disjoint SOP form, where a disjoint SOP form is a form in which the product terms of the function have no overlapping parts in their map representation.

Example 4: The MIGRM form of the set of two functions $F_{1}\left(X_{1}, X_{2}=X_{1}^{023} X_{2}^{01}\right.$, and

$$
\begin{aligned}
F_{2}\left(X_{1}, X_{2}\right) & =X_{1}^{023} X_{2}^{01}+X_{1}^{0} X_{2}^{2} \\
& =X_{1}^{023} X_{2}^{01} \oplus X_{1}^{0} X_{2}^{2}
\end{aligned}
$$

for the polarity used in Examples 1 and 2 is

$$
\begin{aligned}
F_{1} & =X_{1}^{023} X_{2}^{01} \\
& =1 \oplus P_{2}^{3} \oplus P_{1}^{3} \oplus P_{1}^{3} P_{2}^{3} \oplus P_{1}^{4} \oplus P_{1}^{4} P_{2}^{3}
\end{aligned}
$$

as calculated in example 3 , where the map of $F_{1}$ is shown in Fig. 6. The second function $F_{2}$ is composed of the

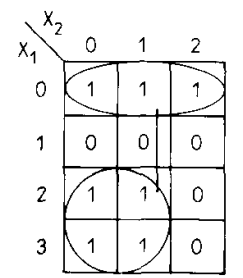

Fig. 6 Map of function $F_{2}\left(X_{1}, X_{2}\right)$

polarity terms

$$
\begin{aligned}
F_{2} & =X_{1}^{023} X_{2}^{01} \oplus X_{1}^{0} X_{2}^{2} \\
& =1 \oplus P_{1}^{3} \oplus P_{1}^{3} P_{2}^{3} \oplus P_{1}^{4}
\end{aligned}
$$

For a comparison of the function $F_{2}$ with the standard trivial functions for the polarities of variables $X_{1}$ and $X_{2}$ shown in Fig. 5, the map of $F_{2}$ is given in Fig. 6. To apply spectral techniques as in example 3 , the notation for the coefficients from Table 3 is used.

The output functions $F_{1}$ and $F_{2}$ are represented by an output term $f_{1} f_{2}$ for each product term. The output term $f_{1} f_{2}$ is given in the right column of Table 4 . Now the spectrum for each term is calculated separately, similar to example 4. Instead of using ' 1 ' as an entry in the spectrum table, the output term for the product term (Table 4) is taken. As will be shown in Section 3.2, the calcu- lation of the spectrum can be performed in one step for all output functions. However, for case of explanation, in Table 5 we assume that the spectrum for each output function is calculated separately. Thus, the spectrum for each term in each output function can be obtained as shown in example 3 , Table 3 . Because the product term

Table 4: Representation of the multioutput function $F\left(X_{1}\right.$. $\left.X_{2}\right)=F\left(F_{1}, F_{2}\right)$

\begin{tabular}{ll}
\hline Product term & $f_{1} f_{2}$ \\
\hline$X_{1}^{023} X_{2}^{01}$ & 11
\end{tabular}

$X_{1}^{0} X_{2}^{2}$

$X_{1}^{023} X_{2}^{01}$ which is present in $F_{1}$ and $F_{2}$ is the same as in example 3 , the results can be taken directly for the spectrum shown in Table 5. The spectrum for the term $X_{1}^{0} X_{2}^{2}$ can be calculated analogously. The results are given in Table 5. The first bit of the entries 11 and 10 in the table stands for the output function $F_{1}$ and the second one for the output function $F_{2}$.

The calculation of the entries in Table 5 can be performed in two different ways:

(i) Each product term is compared with the standard trivial function of each spectral coefficient by the tabular pattern matching method.

(ii) The spectral coefficients are determined directly from the product term. Thus, the entry ' $\ldots$ in Table 5 indicates that no calculation has to be performed for these cells.

The calculation according to (i) is used in our implementation. $P_{1}^{1} P_{2}^{1}, P_{1}^{1} P_{2}^{3}, P_{1}^{3} P_{2}^{1}$, and $P_{1}^{4} P_{2}^{1}$ are identical to 1 , $P_{2}^{3}, P_{1}^{3}$, and $P_{1}^{4}$ because $P_{1}^{1}=P_{2}^{1}=1$. The row 'EXOR' in Table 5 is obtained by exoring the entries (output functions) in every column. Finally, the last row gives the polarity literals for the spectral coefficients having nonzero entry in the 'EXOR' row. The polarity terms in the result row are the same as obtained for the functions $F_{1}$ and $F_{2}$ at the beginning of this example.

Now the polarity literals have to be replaced by their multiple valued literals as shown in example 3 . Thus, we obtain

$$
F_{1}=1 \oplus X_{2}^{2} \oplus X_{1}^{23} \oplus X_{1}^{23} X_{2}^{2} \oplus X_{1}^{123} \oplus X_{1}^{123} X_{2}^{2}
$$

as calculated in example 3 and

$$
F_{2}=1 \oplus X_{1}^{23} \oplus X_{1}^{123} \oplus X_{1}^{23} X_{2}^{2}
$$

\section{Algorithm for calculation of MIGRM form}

The method for the generation of the MIGRM form consists of two basic stages. First, as described in Section 3.1,

\begin{tabular}{|c|c|c|c|c|c|c|c|c|c|c|c|c|}
\hline Term & $M_{P P_{2}^{1}}$ & $M_{\rho_{1} \rho_{2}^{2}}$ & $M_{P \mid \rho_{2}^{3}}$ & $M_{P_{P_{2}^{2}}^{\prime}}$ & $M_{\rho_{1}^{2} \rho_{2}^{2}}$ & $M_{p_{1}^{2} \rho_{2}^{3}}$ & $M_{P_{1}^{3} \rho_{2}^{1}}$ & $M_{P_{1}^{3} \rho_{2}^{2}}$ & $M_{\rho_{P}^{3} \rho_{2}^{3}}$ & $M_{\rho_{1}^{a} \rho_{2}^{1}}$ & $M_{P_{1}^{4} \rho_{2}^{2}}$ & $M_{P_{1}^{4} \rho_{\frac{3}{3}}}$ \\
\hline$X_{1}^{023} X_{2}^{01}$ & 11 & - & 11 & - & - & - & 11 & - & 11 & 11 & - & 11 \\
\hline$X_{1}^{0} X_{2}^{2}$ & - & - & 01 & - & - & - & - & - & - & - & - & 01 \\
\hline $\begin{array}{l}\text { EXOR } \\
\text { result }\end{array}$ & $\begin{array}{r}11 \\
1\end{array}$ & - & $\begin{array}{l}10 \\
P_{1}^{1} P_{2}^{3}\end{array}$ & - & - & - & $\begin{array}{l}11 \\
P_{1}^{3}\end{array}$ & - & $\begin{array}{l}11 \\
P_{1}^{3} P_{2}^{3}\end{array}$ & $\begin{array}{l}11 \\
P_{1}^{4}\end{array}$ & - & $\begin{array}{l}10 \\
P_{1}^{4} P_{2}^{3}\end{array}$ \\
\hline
\end{tabular}
each multiple valued literal of the $m v$ function has to be transformed to a polarity specified by the chosen orthogonal polarity matrix. In the second stage, presented in Section 3.2 , the terms consisting of transformed literals are used to calculate the final MIGRM form. The code for the transformation of a multiple valued literal is chosen in such a way that the second stage of the transformation is not dependent on the chosen polarity for the

Table 5: Spectrum of function $F\left(X_{1}, X_{2}\right)$ 
literal. This approach requires the introduction of the concept of normalised codes.

\subsection{Transformation for one multiple valued literal}

The basic steps of the algorithm for the transformation of a multiple valued literal to its representation of polarity literals in the normalised code will be illustrated by an example. Table 6 shows the transformations for some possible four-valued literals to the polarity used in the previous examples. The first row gives all the possible combinations of the polarity literals. Let us observe that the first four polarity literals are the rows of matrix $P_{1}$ from example 2 . In the first column, some possible literals of the variable $X$ are given. In the respective row for each of these literals the representation by its polarity literals is given, where the binary representation for the value has to be calculated by performing the EXOR operation among the code words that are determined by ' 1 ' in the table (i.e. $X^{0}=P^{1} \oplus P^{4}=1 \oplus P^{4}$, which is denoted by $1111 \oplus 0111=1000$ ). The second row is then created as follows. Its entries for columns $P^{1}, P^{2}$, and $P^{3}$ are the binary representations of the polarity literals from the polarity matrix. All other entries in the second row are created by the bit-by-bit EXOR operation on the $P^{i}$ literals from the column header. Finally the last row of the table gives the normalised code of the spectral coefficients. It represents the combination of the polarity literals $P^{r}$. For instance, 0111 means that variables $P^{2}, P^{3}$ and $P^{4}$ are used. This row is just a binary encoding of the first row.

As mentioned above, the code for the MIGRM representation of one literal is created in such a way, that this normalised code can be used directly to perform the transformation of the total $m v$ function. This can be done by using the same representation of the polarity literals $P^{1}, P^{2}, \ldots$, for every chosen polarity. The code then determines of what polarity literal/s the initial literal is composed of.

Example 5: The literal $X^{0}$ with the internal representation 1000 can be represented by $P^{1} \oplus P^{4}$ which has the normalised code 1001. The new code representing this combination of polarity literals can be derived by the bit-by-bit OR-operation of the code representation of the polarity literals shown in Table 7 .

The normalised code has a ' $l$ ' in its bit representation corresponding to the index of the polarity literal $P^{r}$
Table 7: Code representation of polarity literals

\begin{tabular}{ll}
\hline Normalised code & Polarity literals $P^{r}$ \\
\hline 1000 & $P^{1}$ \\
0100 & $P^{2}$ \\
0010 & $P^{3}$ \\
0001 & $P^{4}$ \\
\hline
\end{tabular}

The algorithm 1 performs the transformation of a multiple valued literal to the normalised code.

Algorithm 1:

Step 1: Generate all possible EXOR combinations of the polarity literals $P^{r}$ (the first row of Table 6) for later comparison with the original $m v$ literal.

Step 2: Compare the binary representation of the mv literal with the binary representation (row 2, Table 6) of the EXOR combination (row 1, Table 6) of Step 1. If these two binary representations are equal, assign to the $m v$ literal its normalised code (shown in the last row of Table 6).

\subsection{Transformation of a multiple valued function}

After the transformation of each original $m v$ literal, as described above, the whole set of multiple valued terms of the function has to be changed to the MIGRM. Our implementation is based on a tabular pattern matching method, where every product term of the input function is compared with the normalised code of the indices of all spectral coefficients.

The basic steps of the algorithm for the tabular pattern matching are explained in the following example.

Example 6: Let us assume a function $G\left(X_{1}, X_{2}, X_{3}\right)$ where the literal $X_{1}$, being three-valued, is represented by three polarity literals $P_{1}^{1}, P_{1}^{2}$ and $P_{1}^{3}$. The second literal $X_{2}$, being four-valued, is represented by the polarity literals $P_{2}^{1}, P_{2}^{2}, P_{2}^{3}$ and $P_{2}^{4}$, and the three-valued literal $X_{3}$ is represented by $P_{3}^{1}, P_{3}^{2}$, and $P_{3}^{3}$. The notation of the spectral coefficients for a spectrum representing such a function $G\left(X_{1}, X_{2}, X_{3}\right)$ is shown in Table 8 . The code shown in Table 8 is obtained by generating all possible combinations of polarity literals from the distinct original $m v$ literals. This means that not more than one polarity literal per original $m v$ literal can occur in the index of a spectral coefficient (i.e. $M_{P_{1}^{2}}^{2} P_{1}^{3}$ cannot occur because both

Table 6: Transformations of some four-valued literals

\begin{tabular}{lllllllllll}
\hline Literal & $P^{1}$ & $P^{2}$ & $P^{3}$ & $P^{4}$ & $P^{1} \oplus P^{2}$ & $P^{1} \oplus P^{3}$ & $P^{1} \oplus P^{4}$ & $P^{2} \oplus P^{3}$ & $P^{2} \oplus P^{4}$ & $P^{3} \oplus P^{4}$ \\
\cline { 2 - 9 } & 1111 & 0101 & 0011 & 0111 & 1010 & 1100 & 1000 & 0110 & 0010 & 0100 \\
\hline$X^{0}$ & & & & & & 1 & & \\
$X^{1}$ \\
$X^{01}$ \\
$X^{12}$ \\
$X^{012}$
\end{tabular}

IEE PROCEEDINGS-E, VOl. I39, No. 6, NOVEMBER 1992 
Table 8: Complete MIGRM spectrum of function $G\left(X_{1}, X_{2}, X_{3}\right)$ and three-valued $X_{3}$

\begin{tabular}{|c|c|c|c|c|c|c|}
\hline$M_{1}$ & $M_{P_{1} \rho_{2}^{1} P_{3}^{1}}$ & $M_{P_{1} P_{2} P_{3}^{2}}$ & $M_{P \mid P_{2}^{1} \rho_{3}^{3}}$ & $M_{\rho_{1} \rho_{2}^{2} \rho_{3}^{1}}$ & $M_{P\left\{\rho_{2}^{2} \rho_{3}^{2}\right.}$ & $\cdots \quad M_{P_{1}^{3} P_{2}^{4} \rho_{3}^{3}}$ \\
\hline Code & 1001000100 & 1001000010 & 1001000001 & 1000100100 & 1000100010 & $\cdots \quad 0010001001$ \\
\hline
\end{tabular}

polarity literals are from the original $m v$ literal $X_{1}$. As one can observe from the binary representation of the index (second row in Table 8), called code, each spectral coefficient consists basically of a combination of three polarity literals, where each polarity literal is taken from a different original $m v$ literal. Because Table 8 has been created for normalised $m v$ literals, the normalised code has to be used for the code in Table 8 also.

The final value of the spectral coefficient $M_{s}$, where $M_{s}$ is any of the possible spectral coefficients, determined by a column in Table 8 , is obtained by comparing the normalised codes of all product terms, further called term $_{x}(x=0, \ldots, m-1$; where $m$ is the total number of terms) of the Boolean function including variables $X_{1}$, $X_{2}, \ldots, X_{n}$ with the representation of the spectral coefficients code from Table 8 . The value $M_{s}$ for term $m_{x}$ is the representation of the output functions $f_{x}$ of term , $_{x}$, if the intersection of the codes and the $\operatorname{term}_{x}$ is not empty. To obtain the final value $M_{s}$ for the whole function (all its terms), the EXOR operation has to be performed among all $m$ values $M_{s}$. The calculation of the values of coefficients $M_{s}$ is described by

$$
\begin{aligned}
M_{s}^{0} & =0 \\
M_{s}^{x+1} & =M_{s}^{x} \oplus\left(\left(\left(\operatorname{code}_{s} \& \text { term }_{x}\right) \neq \varnothing\right) \& f_{x}\right),
\end{aligned}
$$

$$
\text { for } x=0, \ldots, m-1
$$

where the value of the spectral coefficient is a product term, code and $_{\text {term }}$ are the binary representations of the respective literals, where $\left(\left(\right.\right.$ code $_{s} \&$ term $\left.\left._{x}\right) \neq \varnothing\right)$ has to be true for the intersection of each literal of code $e_{s}$ and term $_{x}$. By $\varnothing$ we denote a vector which contains at least one zero. The output product term for term $m_{x}$ is denoted by $f_{x}$.

The final MIGRM form consists of the terms obtained by replacing the polarity literals in the indices of the spectral coefficients $M_{s}$ (row code in Table 8) which have a value that is not ' 0 ', with their binary representation.

To summarise, the procedure for obtaining the MIGRM of a multioutput Boolean function can be described by algorithm 2 :

Algorithm 2:

Step 1: Transform all the multiple valued input literals of the function to their normalised codes according to the chosen polarities for the variables (Section 2, algorithm 1).

Step 2: Calculate the MIGRM spectrum for the normalised codes as presented in Section 2.2.

Step 3: Replace the polarity literals of non-zero spectral coefficients by their original $m v$ literal. The output functions for the terms are given by the values of the spectral coefficients.

As one can observe, each spectral coefficient can be calculated in turn. Thus, it can be stored directly on hard disk. With this approach, the necessity to keep the whole spectrum in the computer memory has been overcome.

\section{Practical example}

A real-life example is included to illustrate the complete MIGRM transformation. The function used is a two-bit

\begin{tabular}{|c|c|c|c|c|c|}
\hline \multirow{2}{*}{$\frac{\text { Binary }}{x_{1}, x_{2}, x_{3}, x_{4}}$} & \multicolumn{2}{|c|}{ Multiple valued } & \multicolumn{2}{|c|}{ Normalised } & \multirow{2}{*}{$\frac{\text { Output }}{f_{c} f_{0} f_{1}}$} \\
\hline & $x_{1}$ & $x_{2}$ & $x_{1}$ & $x_{2}$ & \\
\hline 0000 & 1000 & 1000 & 1110 & 1110 & 000 \\
\hline 0001 & 1000 & 0100 & 1110 & 1111 & 001 \\
\hline 0010 & 1000 & 0010 & 1110 & 0010 & 010 \\
\hline 0011 & 1000 & 0001 & 1110 & 1011 & 011 \\
\hline 0100 & 0100 & 1000 & 1111 & 1110 & 001 \\
\hline 0101 & 0100 & 0100 & 1111 & 1111 & 010 \\
\hline 0110 & 0100 & 0010 & 1111 & 0010 & 011 \\
\hline 0111 & 0100 & 0001 & 1111 & 1011 & 100 \\
\hline 1000 & 0010 & 1000 & 0010 & 1110 & 010 \\
\hline 1001 & 0010 & 0100 & 0010 & 1111 & 011 \\
\hline 1010 & 0010 & 0010 & 0010 & 0010 & 100 \\
\hline 1011 & 0010 & 0001 & 0010 & 1011 & 101 \\
\hline 1100 & 0001 & 1000 & 1011 & 1110 & 011 \\
\hline 1101 & 0001 & 0100 & 1011 & 1111 & 100 \\
\hline 1110 & 0001 & 0010 & 1011 & 0010 & 101 \\
\hline 1111 & 0001 & 0001 & 1011 & 1011 & 110 \\
\hline
\end{tabular}

Table 9: Truth table of two-bit adder

adder (Table 9, where the two four-valued literals $X_{1}$ and $X_{2}$ represent two binary values each $\left(X_{1}=\left(x_{1}, x_{2}\right)\right.$, $\left.X_{2}=\left(x_{3}, x_{4}\right)\right)$.

The input variable assignment is not unique. Thus, there exist many different assignments. In this example, the $m v$ literal $X_{1}$ is obtained by changing the first two bits of the binary function $\left(x_{1}, x_{2}\right)$ to a four-valued literal $\left(X_{1}^{0}=00, X_{1}^{1}=01, X_{1}^{2}=10, X_{1}^{3}=11\right)$. The second two bits are used to obtain $X_{2}$.

To make it easier to follow the steps of the transformation, the same polarity is assumed for both literals. The polarity literals $P_{i}^{r}$ and the binary representations of their values $\left(T_{i}^{r}\right)$ are given in Table 10 .

Table 10: Polarity for two-bit adder

\begin{tabular}{ll}
\hline Polarity for $X_{1}$ & Polarity for $X_{2}$ \\
\hline$P_{1}: 1111$ & $P_{2}^{1}: 1111$ \\
$P_{1}^{2}: 0101$ & $P_{2}^{2}: 0101$ \\
$P_{1}^{3}: 0010$ & $P_{2}^{3}: 0010$ \\
$P_{1}^{4}: 1100$ & $P_{2}^{4}: 1100$ \\
\hline
\end{tabular}

Now the binary representations of the literals $X_{1}$ and $X_{2}$ in Table 7 are replaced by their normalised codes (algorithm 1). Because only four different values occur in the literals $X_{1}$ and $X_{2}$, the normalised codes for those values are shown in Table 11 , where $P^{r}=P_{1}^{r}=P_{2}^{r}$. The

Table 11 : Normalised codes for four occurring values

\begin{tabular}{lll}
\hline $\begin{array}{l}\text { Binary value } \\
\text { of literal }\end{array}$ & Normalised code & $\begin{array}{l}\text { Cube representation } \\
\text { of normalised code }\end{array}$ \\
\hline 0001 & $P^{1} \oplus P^{3} \oplus P^{4}$ & 1011 \\
0010 & $P^{3}$ & 0010 \\
0100 & $P^{1} \oplus P^{2} \oplus P^{3} \oplus P^{4}$ & 1111 \\
1000 & $P^{1} \oplus P^{2} \oplus P^{3}$ & 1110 \\
\hline
\end{tabular}

multiple valued literals $X_{1}$ and $X_{2}$ shown in Table 9 are now substituted with the cube representations of their normalised codes given in Table 11; the result is given in Table 9.

The normalised code obtained in this procedure is now compared with all the indices of the spectral coefficients of the general spectrum for a function $G\left(X_{1}, X_{2}\right)$,

IEE PROCEEDINGS-E.VOI. 139, NO. 6, NOVEMBER 1992 
Table 12: Spectrum for first three terms from table 9

\begin{tabular}{|c|c|c|c|c|c|c|c|c|}
\hline \multirow[t]{2}{*}{ Term } & $M_{P_{1} P_{2}^{1}}$ & $M_{\rho\} \rho_{2}^{2}}$ & $M_{P \mid \rho_{2}^{3}}$ & $M_{\rho_{1}^{1} \rho_{2}^{a}}$ & $M_{\rho_{1}^{2} \rho_{2}^{1}}$ & $M_{\rho_{1}^{2} \rho_{2}^{2}}$ & $M_{\boldsymbol{\rho}_{1}^{x} \boldsymbol{\rho}_{2}^{3}}$ & $M_{\boldsymbol{P}_{1}^{2} P_{2}^{4}}$ \\
\hline & 10001000 & 10000100 & 10000010 & 10000001 & 01001000 & 01000100 & 01000010 & 0100000 \\
\hline \multirow{3}{*}{$\begin{array}{l}11101111 \\
11100010 \\
11101011\end{array}$} & 001 & 001 & 001 & 001 & 001 & 001 & 001 & 001 \\
\hline & - & - & 010 & - & - & - & - & 010 \\
\hline & 011 & - & 011 & 011 & 011 & - & 011 & 011 \\
\hline Result & 010 & 001 & 000 & 010 & 010 & 001 & 010 & 000 \\
\hline \multirow[t]{2}{*}{ Term } & $\underline{M_{P_{1}^{3} P_{2}^{1}}}$ & $M_{\rho_{1}^{3} \rho_{2}^{\frac{2}{2}}}$ & $M_{P_{1}^{3} \rho_{2}^{3}}$ & $M_{P_{1}^{3} P_{2}^{4}}$ & $M_{P_{1}^{4} P_{z}^{1}}$ & $M_{P_{1}^{4} P_{2}^{2}}$ & $M_{P_{1}^{4} P_{2}^{3}}$ & $M_{p_{1}^{4} p_{2}^{A}}$ \\
\hline & 00101000 & 00100100 & 00100010 & 00100001 & 00011000 & 00010100 & 00010010 & 0001000 \\
\hline 11101111 & 001 & 001 & 001 & 001 & - & - & - & - \\
\hline 11100010 & - & - & 010 & - & - & - & - & - \\
\hline 11101011 & 011 & - & $0 \nmid 1$ & 011 & - & - & - & - \\
\hline Result & 010 & 001 & 000 & 010 & 000 & 000 & 000 & 000 \\
\hline
\end{tabular}

where $X_{1}$ and $X_{2}$ are four-valued literals (algorithm 2, step 2). Table 12 illustrates the calculation of the spectrum for the first three terms from Table 9. The Table has the output functions of the terms as entries if, for each term, the intersection of the term and the index of the coefficient is not empty (eqn. 3); otherwise the entry is '- For instance, the cell for the intersection of row 11101011 and column 01001000 is ' $011^{\prime}$ ' as the intersection of those indices is 01001000 where both literals are not empty. The intersection of row 11100010 and column 01001000 is $01000000 \mathrm{so}$ '- ' is placed in the corresponding cell. The final coefficients for the partial function in Table 12 are obtained by exoring the entries of the columns. The result of this operation is given in the last row.

The result of the complete function is shown in Table 13. The first column of Table 13 lists all nonzero spectral

Table 13: MIGRM of 2-bit adder

\begin{tabular}{|c|c|c|c|c|}
\hline $\begin{array}{l}\text { Spectral } \\
\text { coefficient }\end{array}$ & index & $x_{1}$ & $x_{2}$ & $f_{c} f_{0} f_{1}$ \\
\hline$M_{P \mid \rho \frac{1}{2}}$ & 10001000 & 1111 & 1111 & 100 \\
\hline$M_{\rho i \rho_{2}^{2}}$ & 10000100 & 1111 & 0101 & 101 \\
\hline$M_{P \mid p_{2}^{4}}$ & 10000001 & 1111 & 1100 & 110 \\
\hline$M_{P_{1}^{2} p \frac{1}{2}}$ & 01001000 & 0101 & 1111 & 101 \\
\hline$M_{P_{1}^{2} p \frac{z}{2}}$ & 01000100 & 0101 & 0101 & 010 \\
\hline$M_{\rho_{1}^{2} \rho_{\frac{3}{2}}^{2}}$ & 01000010 & 0101 & 0010 & 100 \\
\hline$M_{P_{1}^{2} p_{2}^{4}}$ & 01000001 & 0101 & 1100 & 100 \\
\hline$M_{\rho_{1}^{3} \rho_{2}^{2}}$ & 00100100 & 0010 & 0101 & 100 \\
\hline$M_{P_{1}^{4} P_{2}^{1}}$ & 00011000 & 1100 & 1111 & 110 \\
\hline$M_{p_{1}^{4} p_{2}^{2}}$ & 00010100 & 1100 & 0101 & 100 \\
\hline$M_{p_{1}^{A} \rho_{2}^{A}}$ & 00010001 & 1100 & 1100 & 100 \\
\hline
\end{tabular}

coefficients $M_{s}$ that occur in any of its component functions. These coefficients have been obtained by comparing (as in Table 12) the normalised binary representations of their indices (listed in the second column of Table 13) with the normalised code obtained according to Tables 10 and 11 . Finally, the binary representation for the literals of variables $X_{1}$ and $X_{2}$ is obtained by replacing the polarity literals of the indices of spectral coefficients from the first row, by their binary representations (algorithm 2, step 3). According to Table 10, the conversion is: 1000 to 1111,0100 to 0101,0010 to 0010 , and 0001 to 1100 . The output terms $f_{c} f_{0} f_{1}$ are the values of the spectral coefficients.

The implementation of the above result as an ANDEXOR PLA with two-input, three-output decoders for the chosen polarities is shown in Fig. 7.

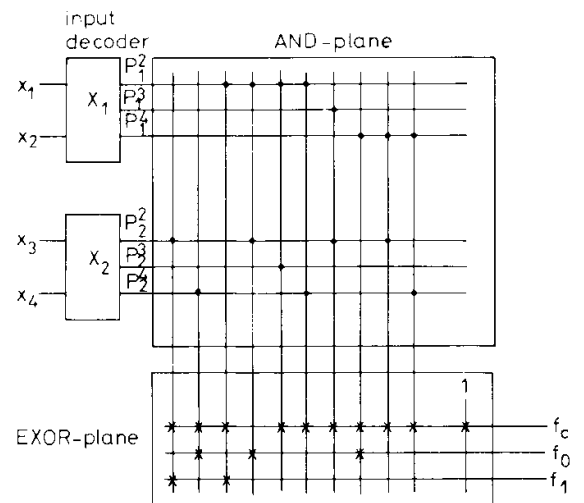

Fig. 7 AND-EXOR PLA implementation with input decoders of the MIGRM form of the 2-bit adder

The input decoders are the same because the same polarity has been chosen for both literals, the input decoder for both variables $X_{1}$, and $X_{2}$ is shown in Fig. 8 .

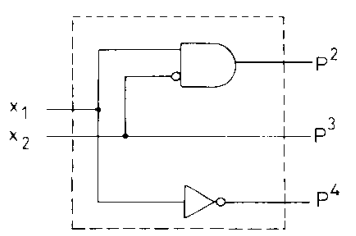

Fig. 8 Input decoder for variables $X_{1}$ and $X_{2}$

\section{Evaluation and results}

The calculation method presented for the computation of the MIGRM has been implemented in the program GRM-MV (generalised Reed-Muller synthesiser, multiple valued version). The numerical results have confirmed the validity of the MIGRM concept. However, the problem of finding a polarity with the minimal number of product terms without the computation of all possible forms still has to be solved. It is known, that for an $n$ variable Boolean function, there are $2^{n}$ GRM forms. For an $n$ variable function where each variable is threevalued, there are $28^{n}$ forms, and for each variable being four-valued there are $840^{n}$ forms. Therefore, the computation of the form having the minimal number of product terms by going through all forms is very complex. Moreover, the pairing of the Boolean variables to obtain the 
multiple valued form also has an impact on the number of necessary product terms in the MIGRM.

For the evaluation of the MIGRM in comparison to the GRM, the number of polarities for a four-valued variable has been restricted to 60 randomly selected polarities. Therefore, the total number of polarities considered was $60^{n}$. Table 14 gives the results obtained for some MCNC benchmark functions.

Table 14: Benchmark results for MIGRM

\begin{tabular}{lrrrr}
\hline & ESPRESSO & GRM & $\begin{array}{l}\text { ESPRESSO } \\
\text { 2-bit decoder }\end{array}$ & $\begin{array}{l}\text { MIGRM } \\
\text { 2-bit decoder }\end{array}$ \\
\hline adr2 & 11 & 8 & 9 & 5 \\
adr4 & 75 & 34 & 66 & 34 \\
bw & 22 & 22 & 23 & 22 \\
con1 & 9 & 17 & 9 & 17 \\
inc & 30 & 47 & 28 & 40 \\
rd53 & 31 & 20 & 12 & 15 \\
rd73 & 127 & 63 & 37 & 40 \\
rd84 & 255 & 107 & 54 & 54 \\
squar5 & 25 & 23 & 26 & 25 \\
xor5 & 16 & 5 & 4 & 4 \\
5xp1 & 65 & 61 & 55 & 64 \\
\hline
\end{tabular}

The column ESPRESSO lists the number of product terms obtained by the two-level SOP optimizer ESPRESSO [13]. The following column gives the minimal number of product terms in the respective GRM form For the computation of the MIGRM and MIESOP based on four-valued variables, pairs of Boolean variables have been taken to obtain a four-valued one. The results obtained are given in the last columns of Table 14 .

One can observe from the obtained results that the MIGRM gives a reduction of up to $50 \%$ in the number of product terms over the GRM. Moreover, the number of product terms of the GRM is the absolute minimum while only a local minimum is obtained for the MIGRM. This is due to the restriction of the number of polarities per variable to 60 instead of 840

The results obtained give motivation to further investigate in MIGRM forms. However, methods to avoid the computation of all possible polarities similar to the one introduced for GRMs [5,25] have to be developed to overcome the high computational complexity of MIGRM forms. Additionally, it has to be investigated which multiple valued decoders are practical for circuit realisations. Then, the search for the minimal MIGRM form can be restricted to the one which is optimal with respect to a certain set of multiple valued decoders.

\section{Conclusions}

The extension of the general Reed-Muller expansion to multiple valued input, binary multioutput functions has been shown. For this, the concept of code normalisation of single multiple valued literals to perform a final transformation has been developed. The code normalisation is applied to make the transformation of the complete function independent of the polarity chosen. This simplifies and speeds up the main transformation step to the final MIGRM form for the transformed single $m v$ literal. For further investigations of the properties of such forms, the MIGRM transformation has been implemented as a computer algorithm. Because an exhaustive search of all $\prod_{i=0}^{n} p\left(X_{i}\right)$ MIGRM forms, where $p\left(X_{i}\right)$ is the number of possible polarities for variable $X_{i}$ with $i=1, \ldots, n$, would be too time consuming, further research has to be concentrated on avoiding a complete search and to finding immediately good polarities as has been carried out for GRM forms $[5,25]$.

As circuits which realise the MIGRM forms are both easily testable, or modifiable to very easily testable circuits, further research into them is important. It must however, be experimentally found with practical logic benchmarks how much of a circuit cost penalty we pay with respect to the corresponding MIESOPs. The role of input variable pairing [15] must also be investigated, as a good pairing together with a good choice of variable polarities may significantly improve the cost.

\section{References}

1 AKERS, S.B.: 'On a theory of Boolean functions', SIAM J., 1959, 7 , pp. $487-498$

2 GREEN, D.H.: 'Modern logic design' (Electronic Systems Engineering Series, 1986)

3 DAVIO, M., DESCHAMPS, J.P., and THAYSE, A.: 'Discrete and switching functions' (MoGraw-Hill, 1978)

4 GREEN, D.H.: 'Families of Reed-Muller canonical forms', Int. J Electron', 1991, 63, (2), pp. 259-280

5 SARABI, A., and PERKOWSKI, M.A.: 'Fast exact and quasiminimal minimization of highly testable fixed-polarity AND/XOR canonical networks'. Proceedings of the 29 th Design Automation Conference, Anaheim, CA, June 1992, pp. 30-35

6 BHATTACHARYA, B.B., GUPTA, B., SARKAR, S., and 6 BHATTACHARYA, B.B., GUPTA, B., SARKAR, S., and
CHOUDHURY, A.K.: 'Testable design of RMC networks with universal tests for detecting stuck-at and bridging faults', IEE Proc. $E$, Comput. Digit. Tech., 1985, 132, (3), pp. 155-161

7 DAMARLA, T., and KARPOWSKY, M.: 'Detection of stuck-at and bridging faults in Reed-Muller canonical (RMC) networks', IEE Proc. E. Comput. Digit. Tech., 1989, 136, (5), pp. 430-433

8 KODANDAPANI, K.L.: 'A note on easily testable realizations for KODANDAPANI, K.L.: 'A note on easily testable real

9 PRADHAN, D.K.: 'Universal test sets for multiple fault detection in AND-EXOR arrays', IEEE Trans. Comput., 1978, 27, (2), pp. 181 187

10 REDDY, S.M.: 'Easily testable realizations for logic functions', IEEE Trans. Comput., 1972, 21, (11), pp. 1182-1188

11 SALUJA, K.K., and REDDY, S.M.: 'Fault detecting test sets for Reed-Muller canonic networks', IEEE Trans. Comput., 1975, 24, (10), pp. 995-998

12 REDDY, B.R.K., and PAI, A.L.: 'Reed-Muller transform image coding', Comput. Vis., Graph. Image Process., 1988, 42, pp. 48-61

13 RUDELL, R., and SANGIOVANNI-VINCENTELLI, A. 'Multiple-valued minimization for PLA optimization', IEEE Trans. Comput.-Aided Des. Integrated Circuits and Syst., 1987, 6, (5). pp. $727-750$

14 SASAO, T.: 'On the optimal design of multiple-valued PLA's', IEEE Trans. Comput., 1989, 38, (4), pp. 582-592

15 SASAO, T.: 'Multiple-valued decomposition of generalized Boolean functions and the complexity of programmable logic arrays', IEEE functions and the complexity of programmat
Trans. Comput., 1981, 30, (9), pp. 636-643

16 BRAYTON, R.K., RUDELL, R,, SANGIOVANNIVINCENTELLI, A., and WANG, A.R.: 'MIS: multi-level interactive logic optimization system', IEEE Trans., Comput.-Aided Des. Integrated Circuits and Syst., 1989, 6, (6), pp. 1062-1082

17 SASAO, T.: 'MACDAS: multi-level AND-OR circuit synthesis using two-variable function generators', Proceedings of the $23 \mathrm{rd}$ Design Automation Conference, June 1986, pp. 86-93

18 PERKOWSKI, M.A., HELLIWELL, M., and WU, P.: 'Minimization of multiple-valued input multi-output mixed-radix exclusive sums of products for incompletely specified Boolean functions' 19 th Int. Symp. on Multiple-Valued Logic, May 1989, pp. 256-263

19 SCHÄFER, I., and PERKOWSKI, M.A.: "Multiple valued generalized Reed-Muller forms', 21st Int. Symp. on Multiple-Valued Logic, May 1991, pp. 40-48

20 SASAO, T.: 'EXMIN: a simplification algorithm for exclusive-ORsum-of-products expressions for multiple-valued input two-valued output functions', 20th Int. Symp. on Multiple-Valued Logic, May output functions',
1990, pp. 128-135

$21 \mathrm{HU}, \mathrm{Z}$.: 'The simple methods for evaluating the coefficients of the canonical RM expansion of multivalued functions', Int. J. Electron. $1987,63,(6)$, pp. $851-856$

22 KODANDAPANI, K.L., and SETLUR, R.V.: 'Reed-Muller canonical forms in multivalued logic', IEEE Trans. Comput., 1975, pp. 628-636

23 FALKOWSKI, B.J., and PERKOWSKI, M.A.: 'Walsh type transforms for completely and incompletely specified multiple-valued

IEE PROCEEDINGS-E, Vol. 139, No.6, NOVEMBER 1992 
input binary functions', 20th Int. Symp. on Multiple-Valued Logic, May 1990, pp. $75-82$

24 FALKOWSKI, B.J., and PERKOWSKI, M.A.: 'On the calculation of generalized Reed-Muller canonical expansions from Disjoint cube representation of Boolean functions'. IEEE 33rd Midwest

Symp. on Circuits \& Systems, August 1990, pp. 1131-1133

5 ALMAINI, A.E.A.: 'Tabular techniques for Reed-Muller logic', Int. J. Electron., 1991, 70, (1), pp. 23-34 